\title{
Microtubule Fragmentation and Partitioning in the Axon during Collateral Branch Formation
}

\author{
Wenqian Yu, Fridoon J. Ahmad, and Peter W. Baas \\ Department of Anatomy, The University of Wisconsin Medical School, Madison, Wisconsin 53706
}

\begin{abstract}
Axons within the brain branch principally by the formation of collaterals rather than by bifurcation of the terminal growth cone (O'Leary and Terashima, 1988). This same behavior is recapitulated in cultures of embryonic hippocampal neurons (Dotti et al., 1988), rendering them ideal for studies on the cell biological mechanisms underlying collateral branch formation. In the present study, we focused on changes in the microtubule (MT) array that occur as these axons branch. In particular, we explored the mechanism by which MT number is locally increased to accommodate the need for more MTs during collateral branch formation. Serial reconstruction analyses indicate that MT number increases by severalfold and that MT length decreases correspondingly within the parent axon in the discrete region giving rise to the branch. These observations strongly suggest that MTs within the parent axon undergo a local fragmentation in this region, and hence raise the possibility that a portion of these new MTs might be destined for transport into the branch. To address this latter issue, we used quantitative immunofluorescence to compare the proportion of newly assembled to total MT polymer in different regions of the axon. As previously reported (Brown et al., 1992), the region of the axon contiguous with the terminal growth cone is particularly rich in newly assembled polymer. In contrast, there was no distinguishable difference in the proportion of newly assembled polymer in the newly formed collateral branches compared to the shaft region of the parent axon. These results indicate that the MTs within the newly formed collateral branches are on average assembled at the same time as those within the parent axon, and thus strongly suggest that the MTs in the collateral branch were assembled in the parent axon and then translocated into the branch. We conclude on the basis of these observations that collateral branch formation requires a local fragmentation of MTs within the parent axon, followed by the partitioning of a portion of the MT fragments into the branch. These short MTs presumably then resume their movement and elongation down the collateral branch as well as down the parent axon for the steady and orderly increase of both MT arrays.
\end{abstract}

\footnotetext{
Received Feb. 16, 1994; revised Mar. 31, 1994; accepted Apr. 21, 1994.

We thank Drs. Katherine Kalil and Mei Lu (both of the University of Wisconsin, Madison) for helpful discussions on axon branching, and for their critical comments on the manuscript. This work was funded by National Institutes of Health Grant NS 28785 and National Science Foundation Grant IBN-9209939 to P.W.B., who is also the recipient of a Research Career Development Award from the National Institutes of Health.

Correspondence should be addressed to Dr. Peter W. Baas, Department of Anatomy, The University of Wisconsin Medical School, 1300 University Avenue, Madison, WI 53706.
}

Copyright $(\mathcal{C} 1994$ Society for Neuroscience $0270-6474 / 94 / 145872-17 \$ 05.00 / 0$
[Key words: hippocampal neuron, axon, collateral branching, microtubule, microtubule fragmentation, microtubule transport, microtubule assembly]

The complexity of the nervous system is dependent upon the elaboration of highly elaborate and exquisitely organized axonal arbors. Most neurons within the brain extend only one axon, but individual axons can branch extensively to increase by manyfold their cross-sectional area and number of axon terminals available for synapse formation. Observations on axon branching in the brain indicate that branches form principally as collaterals along the length of the axon rather than by growth cone bifurcation (O'Leary and Terashima, 1988). Beyond this, however, little is known about the cell biological mechanisms by which axon branching occurs. For example, little is known about the structural changes that must occur in the axon in order for a branch to form. In addition, it is unknown how the pattern of cytoplasmic organization within the parent axon is passed on to each new branch. Of special interest in this regard is the microtubule (MT) system of the axon, an assembly of highly organized filaments that are essential for defining the architecture and cytoplasmic composition of the axon. In the present study, we have sought to elucidate changes in the MT array of the axon that occur during the formation of collateral branches. In particular, our goal was to understand how the highly organized MT pattern of the parent axon is recapitulated within the collateral branches as they form.

Within the axon, the MT array is continuous from the cell body into the terminal growth cone, but individual MTs vary in length, stopping and starting at various points within the array (Bray and Bunge, 1981; Yu and Baas, 1994). All of the MTs have a consistent 13-protofilament lattice (Tilney, 1973; Burton et al., 1975), and are uniformly oriented with regard to their intrinsic polarity, with plus ends directed away from the cell body (Heidemann et al., 1981; Baas et al., 1988). Although it has been long recognized that axonal M'Ts are not attached to any discernable nucleating structure such as the centrosome (Lyscr, 1968), contemporary studics suggest that axonal MTs are in fact nucleated at the centrosome, after which they are released for transport into the axon (Baas and Joshi, 1992; Baas and Ahmad, 1993; Yu et al., 1993; Ahmad et al., 1994). This view is attractive in that centrosomal nucleation would ensure the consistent lattice structure of the MTs, and orderly transport by a plus-end-leading transport motor would ensure their uniform polarity orientation. Additional data strongly suggest that no new MTs are nucleated within the axon itself (Baas and Heidemann, 1986; Baas and Ahmad, 1992), thus preserving the high degree of organization and consistent lattice structure resulting from orderly transport and centrosomal nucleation. 
Although attractive, these ideas present a dilemma with regard to the formation of collateral branches. As a collateral branch forms, it requires a generous supply of highly organized MTs, and these requirements may approach or even exceed those of the parent axon itself. Moreover, the demand for more MTs is heightened exponentially by the fact that a parent axon may extend several collateral branches, and each collateral branch itself may then act as a parent axon and extend its own collateral branches. Notably, collateral branches form at sites many hundreds of micrometers or more from the cell body, and it is difficult to imagine how the tremendous number of additional MTs required for branch formation could derive directly from the centrosome and translocate at necessary rates over such great distances. It seems more likely that local mechanisms exist within the axon to account for the rapid and focal increase in MI number required to supply the formation and growth of collateral branches. In light of these considerations, we have proposcd that MTs in the axon, in response to appropriate stimuli, can locally fragment (Joshi and Baas, 1993). Such fragmentation would transform one MT into many, all of which would be assembly competent and capable of rapidly elongating, and all of which would inherit the centrosomally derived characteristics of their predecessor MTs. In support of such a possibility, recent work on non-neuronal cells and cell extracts suggests that the severing of MTs into fragments may be physiologically relevant in a number of different biological systems (Sanders and Salisbury, 1989; Vale, 1991; Febvre-Chavalier and Febvre, 1992; Shiina et al., 1992; McNally and Vale, 1993).

In the present study, we have sought to investigate the merits of our proposal by studying axon branching in cultures of embryonic rat hippocampal neurons. Similar to axons within the brain, the axons in these cultures branch principally by the formation of collaterals (Dotti et al., 1988), rendering them appropriate and useful for studies on the cell biological mechanisms by which collateral branches form. We explored changes in MT lengths and organization that occur as a parent axon generates a collateral branch, and compared the age of the MT polymer within the newly formed branches with that of the polymer within the parent axon. The latter analyses were aimed at determining whether the polymer within a newly formed branch is all newly assembled, or whether a portion of the polymer within the branch is assembled in the parent axon and translocated into the branch during its formation.

\section{Materials and Methods}

Cell culture. Cultures of embryonic rat hippocampal neurons were preparcd as previously described (Goslin and Banker, 1991). Briefly, hippocampi were dissected from $18 \mathrm{~d}$ rat embryos, treated with trypsin for $15 \mathrm{~min}$, dissociated with trituration, and plated at a density of $\approx 1000$ cells $/ \mathrm{cm}^{2}$ in Minimum Essential Medium (GIBCO-Bethesda Research Labs, Grand Island, NY) containing 10\% horse serum. The cells were plated onto either plastic tissue culture dishes or glass coverslips. Cells on the plastic substratum were used for live cell observations and electron microscopy, while cells grown on glass coverslips were used for immunofluorescence work. In either case, the glass or plastic had been treated with polylysine and rinsed extensively prior to plating the cells. After $4 \mathrm{hr}$ of attachment, a glass coverslip containing a monolayer of astroglial cells was placed on top of the neurons growing on plastic, while the glass coverslips containing neurons were inverted into plastic dishes containing astroglial cells. At this point, the medium consisted of Minimum Essential Medium, the N2 supplements described by Bottenstein (1985), $1 \mathrm{~mm}$ sodium pyruvate, and $0.1 \%$ ovalbumin. Cytosine arabinoside was added to the cultures on the third day to a final concentration of $5 \times 10^{-6} \mathrm{M}$ to inhibit the proliferation of non-neuronal cells. Experiments were performed on cultures that were at least 1 week but not greater than 2 weeks old. By this time, all of the neurons had clearly entered stage 4 of development as defined by Dotti et al. (1988), and the axons had grown into an extensive and complex network.

Live cell observations. For visualization of collateral branch formation, regions of cultures containing axons were selected and circled on the bottom of the petri dish using a diamond marker objective so that the same axons could be relocated at subsequent time points. During selection, circling, and photography, the cultures were placed on the stage of a Zeiss Axiovert 35 microscope equipped with a heated stage (Carl Zeiss Incorporated, Thornwood, NY). Despite the advantage of temperature maintenance, work on the microscope stage was performed as rapidly as possible to avoid problems resulting from $\mathrm{pH}$ fluctuations. Cells were visualized using the Zeiss $40 \times$ LD Achroplan objective, which is equipped with a collar to adjust for the thickness of the plastic petri dish. For each axon region of interest, the collar was adjusted to maximize resolution of the finest processes. In most studies, axon regions were photographed on the seventh day after plating, the following morning, and thereafter at $4 \mathrm{hr}$ intervals throughout the second day. In other studies, axon regions were observed over times ranging from minutes to hours.

Electron microscopy. In preparation for electron microscopy, the neuron cultures were fixed at $37^{\circ} \mathrm{C}$ in $0.1 \mathrm{M}$ cacodylate containing $2 \%$ glutaraldehyde, and processed as previously described (Yu and Baas, 1994; modified from Banker and Goslin, 1991). Briefly, after $20 \mathrm{~min}$ of fixation, the cultures were rinsed twice for $5 \mathrm{~min}$ each in $0.1 \mathrm{M}$ cacodylate, postfixed for $5 \mathrm{~min}$ in $1 \% \mathrm{OsO}_{4}$, rinsed twice for $2 \mathrm{~min}$ in $\mathrm{NaCl}$, rinsed twice for $2 \mathrm{~min}$ in water, contrasted for $30 \mathrm{~min}$ in $5 \%$ aqueous uranyl acetate, dehydrated in ethanols, and embedded in LX-112 (Ladd, Burlington, VT). After curing overnight at $60^{\circ} \mathrm{C}$, the tissue-culture plastic on which the cells had been grown was removed from the resin either by tapping with a hammer or by exposure to liquid nitrogen. For enhancement of their light microscopic appearance, embedded cultures were stained at $60^{\circ} \mathrm{C}$ for $30 \mathrm{~min}$ with $1 \%$ toluidine blue. Cells of interest were circled with a diamond marker objective and their images were recorded using a videoprinter (Sony Corporation, Japan). Thin sections with a uniform thickness of $100 \mathrm{~nm}$ were obtained with an Ultracut $\mathrm{S}$ Ultramicrotome (Reichert-Jung, Vienna), picked up on Formvar-coated slot grids, stained with uranyl acetate and lead citrate, and observed with a CX100 electron microscope (JEOL USA, Inc., Peabody, MA). Overlapping photographs at a magnification of $14,000 \times$ were obtained of each section through the axon region of interest.

Because the ultramicrotomy described above fractionates individual MTs within the axon among many thin sections, complete analyses of the MT array within regions of interest require serial reconstruction. To accomplish this, we used recently described modifications ( $\mathrm{Yu}$ and Baas, 1994) of our standard method (Baas and Heidemann, 1986; Joshi et al., 1986; Baas and Joshi, 1992). All MT profiles appearing within the region of interest were traced from the electron micrographs onto transparent cellophane sheets together with membranous borders and some internal membranous elements that acted as registration markers. Ends of MT profiles appearing in each section were assigned coordinates, based on their distance from the registration markers. These coordinates were then used to trace individual MTs through progressive sections. MT profiles in two different sections were judged to be part of the same MT when the proximal end of one MT profile and the distal end of the other MT profile were spaced within two MT diameters $(\approx 0.05 \mu \mathrm{m})$ of one another with regard to all axes. In relatively rare $(<5 \%)$ cases of ambiguity regarding whether two MT profiles were parts of the same or different MTs, the MT profiles were scored as part of the same MT. True composite reconstructions were not prepared because substantial overlap of MTs resulted in ambiguity. Rather, the length, position, and curvature of each MT or MT region were tabulated, and a schematic illustration was prepared to represent the serially reconstructed MT array. It should be noted that this technique suffers in that sample number is necessarily limited by practicality. For this reason serial sections from other axons were examined to ensure that conclusions drawn from the serial reconstructions were generally applicable to comparable regions from other axons.

In other analyses, estimates of the cross-sectional number of MTs traversing discrete regions of the axon were obtained by an abbreviated method. The number of MTs in a single section intersecting a line perpendicular to the long axis of the axon region was counted. This was done thrice over a distance of less than $1 \mu \mathrm{m}$, and the average of the three numbers was calculated. This number was then multiplied by a number equal to one fewer than the total number of sections comprising the width of the axon. The section scored was always in the middle region of the axon, and the number of sections was reduced by one to 


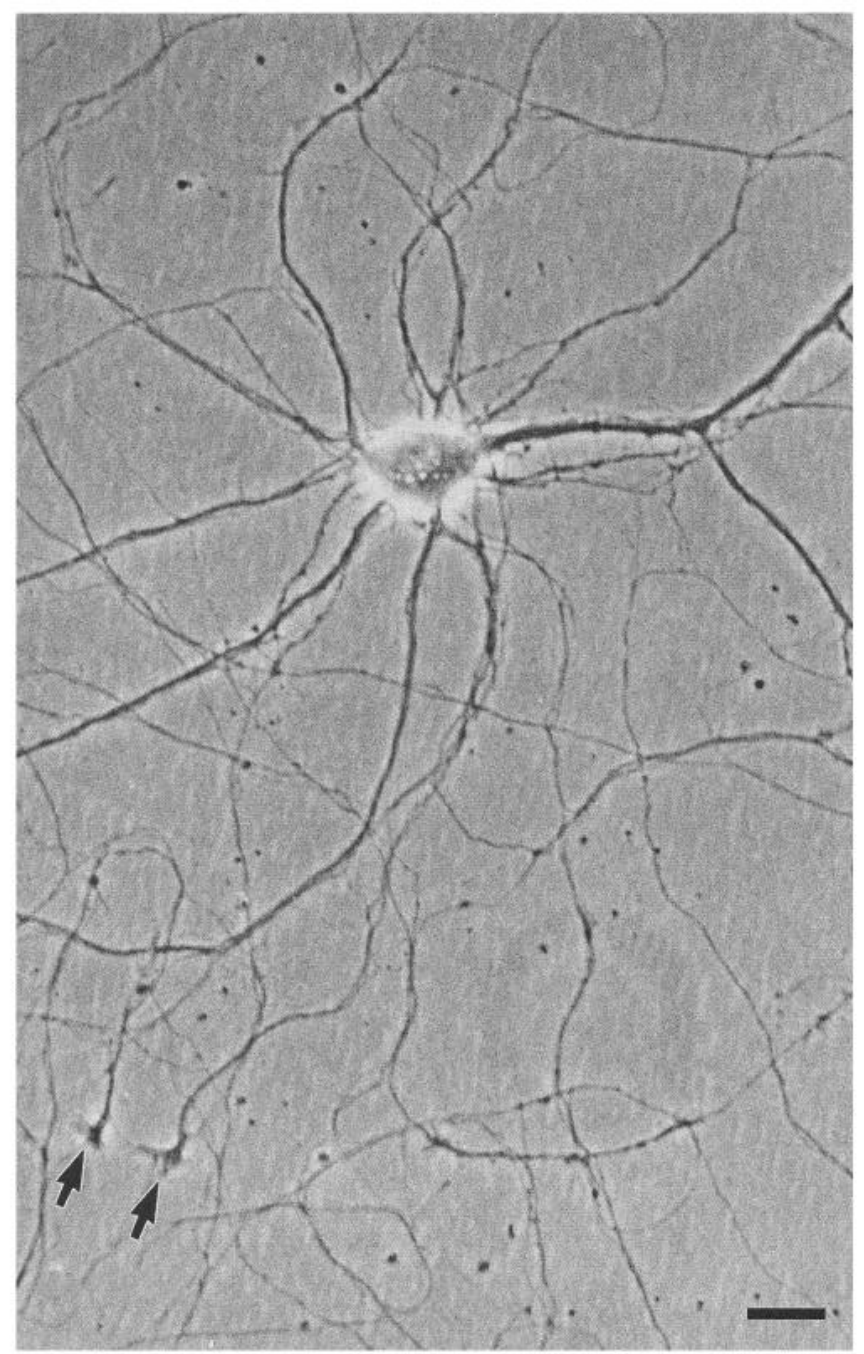

Figure 1. Phase-contrast micrograph of a region of embryonic rat hippocampal neurons in culture for $7 \mathrm{~d}$. The axons have become highly branched and highly fasciculated, resulting in a complex arbor. Typical of a culture of this age, more thickened dendritic processes are also apparent. Axonal growth cones are identifiable (arrows), but it is impossible in a culture of this complexity to trace individual axons from the cell body to their growth cones. Scale bar, $15 \mu \mathrm{m}$.

adjust for the fact that the uppermost and lowermost sections contain fewer MTs compared to the others.

Preparation for immunofluorescence microscopy. Cultures were prepared for immunofluorescence microscopy by a modification of the method of Brown et al. (1992). Briefly, cultures grown on glass coverslips were rinsed in an MT-stabilizing buffer termed PHEM (60 mM PIPES, $25 \mathrm{~mm}$ HEPES, $10 \mathrm{~mm}$ EGTA, $2 \mathrm{~mm} \mathrm{MgCl}_{2}, \mathrm{pH}$ 6.9), and then extracted for $5 \mathrm{~min}$ in PHEM supplemented with $10 \mu \mathrm{m}$ taxol (gift from the National Cancer Institute) and 1\% saponin. Following extraction, cultures were fixed and processed in one of two ways, both of which gave similar results. In the first method, cultures were fixed by the addition of an equal quantity of PHEM containing $8 \%$ paraformaldehyde and $0.3 \%$ glutaraldehyde. After $10 \mathrm{~min}$, cultures were rinsed in PBS, postextracted in $0.1 \%$ Triton X-100 in PBS for $15 \mathrm{~min}$, and then, to reduce autofluorescence, treated three times for $5 \mathrm{~min}$ each in $10 \mathrm{mg} / \mathrm{ml}$ sodium borohydride in PBS. In the second method, the extraction buffer was aspirated from the cultures, after which the coverslips were plunged into $20^{\circ} \mathrm{C}$ methanol (HPLC grade, Aldrich Chemical Company, Milwaukee, WI) for $6 \mathrm{~min}$, and then rehydrated three times for $5 \mathrm{~min}$ in PBS. All cultures, regardless of whether they were fixed with methanol or aldehydes, were then incubated for $30 \mathrm{~min}$ in a blocking solution containing $1 \%$ bovine serum albumen and $2 \%$ normal goat serum in PBS, incubated overnight at $4^{\circ} \mathrm{C}$ with primary antibodies diluted in blocking solution, rinsed three times for 5 min each with PBS, incubated again with blocking solution for $30 \mathrm{~min}$, incubated for $1 \mathrm{hr}$ at $37^{\circ} \mathrm{C}$ with second antibodies, rinsed four times for 5 min each with PBS, and mounted in a medium containing $90 \%$ glycerol, $100 \mathrm{mg} / \mathrm{ml}$ DABCO, $1 \mathrm{mg} / \mathrm{ml}$ phenylenediamine, and $10 \%$ PBS. The primary antibodies were YL $1 / 2$ (purified form purchased from Accurate Chemical and Scientific Corporation, Westbury, NY), a rat monoclonal antibody that recognizes the tyrosinated but not the detyrosinated form of $\alpha$-tubulin (Kilmartin et al., 1982; Wheland et al., 1983), and a mouse monoclonal antibody purchased from Amersham Corporation (Arlington Heights, IL) that recognizes all forms of $\beta$-tubulin. YL $1 / 2$ was used at 1:100, and was visualized with a Texas red-conjugated goat anti-rat second antibody. The $\beta$-tubulin antibody was used at 1:300, and was visualized with a fluorescein-conjugated goat anti-mouse second antibody. Both of the second antibodies, purchased from Jackson Immunoresearch (West Grove, PA), had been preadsorbed to reduce cross-reactivity, and were used at 1:100.

Quantitative immunofluorescence analyses. Cultures prepared for immunofluorescence microscopy were visualized and images were captured using the Zeiss LSM 410 Laser Confocal Microscope. Images were obtained using the Zeiss $63 \times$ PlanAPOCHROMAT objective, and the pinhole on the confocal system was opened maximally to ensure that the entire width of the axon was captured. The instrument is equipped with a krypton/argon laser, and can simultaneously capture with a single scan the Texas red and fluorescein images in perfect alignment. In addition, the LSM 410 has an automated system for producing a third image, each pixel of which represents the ratio of the fluorescence intensity of the Texas red and fluorescein images. In a preliminary experiment, automatic brightness and contrast functions were used to obtain the best possible image of a newly formed collateral branch (in terms of signal to noise and contrast), after which these same settings were used to capture all subsequent images. To produce a ratio image of sufficient contrast, the LSM 410 enables the user to select empirically a factor by which each ratio is uniformly multiplied, and to select a subtraction factor to correct for the contribution of background fluorescence. Again, after maximizing these settings for an image containing collateral branches, the same settings were used for all subsequent work. The original Texas red and fluorescein images together with the ratio image were then transferred to a Macintosh Quadra 800 equipped with the NIH IMAGE software package (provided free of charge from the National Institutes of Health, Bethesda, MD). NIH IMAGE permits fluorescence intensity to be expressed in pseudocolor, in which deep red corresponds to the highest value, light violet corresponds to the lowest, and the intervening color scale corresponds to intervening fluorescence intensity values. The NIH IMAGE software assigns numerical values of $0-255$ ranging from black to white. After defining the portion of the scale covered by our samples, this range was adjusted so that the maximum value was 100 . The same scale was used for all images (i.e., the same color represents the same fluorescence intensity on all ratio images) so that comparison of one image to another would be meaningful.

\section{Results}

\section{Changes in microtubule organization and number at branch points}

The MT array of the axon increases profoundly as the axon branches into a complex arbor. In an initial set of studies, we wished to assess the magnitude of this expansion, and document the changes in MT organization that occur at sites of expansion, namely the branch points. Figure 1 shows a phase-contrast image of a culture of embryonic hippocampal neurons at stage 4 . One cell body with multiple dendrites is apparent, as is a complex network of axons composed of portions of axon arbors from many different neurons. Within the network, the axons are both highly branched and highly fasciculated, resulting in some difficulty in identifying branch points in denser regions of the culture. Fortunately, however, branch points are readily identifiable in electron micrographs, which are also required to visualize the MTs. Electron micrographs from three different axons are presented in Figure 2, each showing a portion of a well-established branch whose total length exceeds $50 \mu \mathrm{m}$. Each micrograph shows four key regions of interest, namely, the 
branch, the proximal and distal sides of the parent axon, and the branch point. The parent axon gives rise to the branch. The proximal and distal sides of the parent axon are the terms we have assigned to the sides of the parent axon flanking the branch directed toward the cell body and terminal growth cone, respectively. The branch point is the centralized transition region joining the proximal side of the parent axon with the distal side of the parent axon and the branch.

As previously reported, the MTs within the parent axon are aligned paraxially and are relatively evenly spaced at about $\approx 50$ $\mathrm{nm}$ apart (Yu and Baas, 1994). With respect to these features of their organization, MTs within branches are entirely similar to the parent axon, which is expected given that collateral branches are often themselves parent axons, giving rise to new collaterals (see introductory remarks). In addition, MT polarity orientation is uniformly plus-end distal everywhere we have looked within the axon arbor (Baas et al., 1988, 1989), indicating that parent axons and collateral branches are identical in this regard as well. At the branch point, a portion of the MTs within the proximal side of the parent axon curves into the branch, while the remaining MTs traverse directly into the distal side of the parent axon. Full appreciation for this partitioning of MTs requires examination of multiple sections through an individual branch point. Some sections show more MTs curving into the branch, and others show more MTs coursing into the distal side of the parent axon (compare Fig. 2a,b). Particularly worth noting is the fact that in all cases, the curved MTs extend into the branch from the proximal side of the parent axon, and never from the distal side. A somewhat unusual case is shown in Figure $2 d$, in which the curved MTs from the proximal side of the parent axon actually overshoot the branch point, extend into the distal side of the parent axon, and then curve back into the branch. In all cases, the number of MTs curving into the branch summed with those going directly into the distal side of the parent axon is greater than the number of MTs within the proximal side of the parent axon, indicating that a local increase in MT number occurs at each branch point.

To assess with more rigor the magnitude by which the MT array increases at the branch point, we obtained estimates for the cross-sectional number of MTs within the proximal and distal sides of the parent axon, and within the branch. The procedure for obtaining these estimates is described in Materials and Methods, and data were obtained for portions of seven different axon arbors, five of which are shown in Figure 3. The cross-sectional number of MTs within the proximal and distal sides of the parent axon were in almost all cases similar to one

Figure 2. Transmission electron micrographs of regions of axons from complex arbors. Each panel shows a branch point, with the proximal and distal sides of the parent axon directed to the left and right, respectively, and the branch directly downward. All branches shown in this figure were well established, measuring at least $50 \mu \mathrm{m}$ in length. At all branch points examined, some MTs could be observed curving into the branch from the parent axon, and this curvature always occurred from the proximal side of the parent axon, and never from the distal side. $a$ and $b$ show different sections through the same branch point. In $a$, a group of MTs traversing directly from the proximal to the distal side of the parent axon are apparent, while the MTs curving into the branch are less obvious. In contrast, $b$ shows several MT profiles curving from the proximal side of the parent axon into the branch, some cutting across other relatively straight MTs. The branch point in $c$ is similar to that shown in $a$ and $b$, with curved MTs directed into the branch and
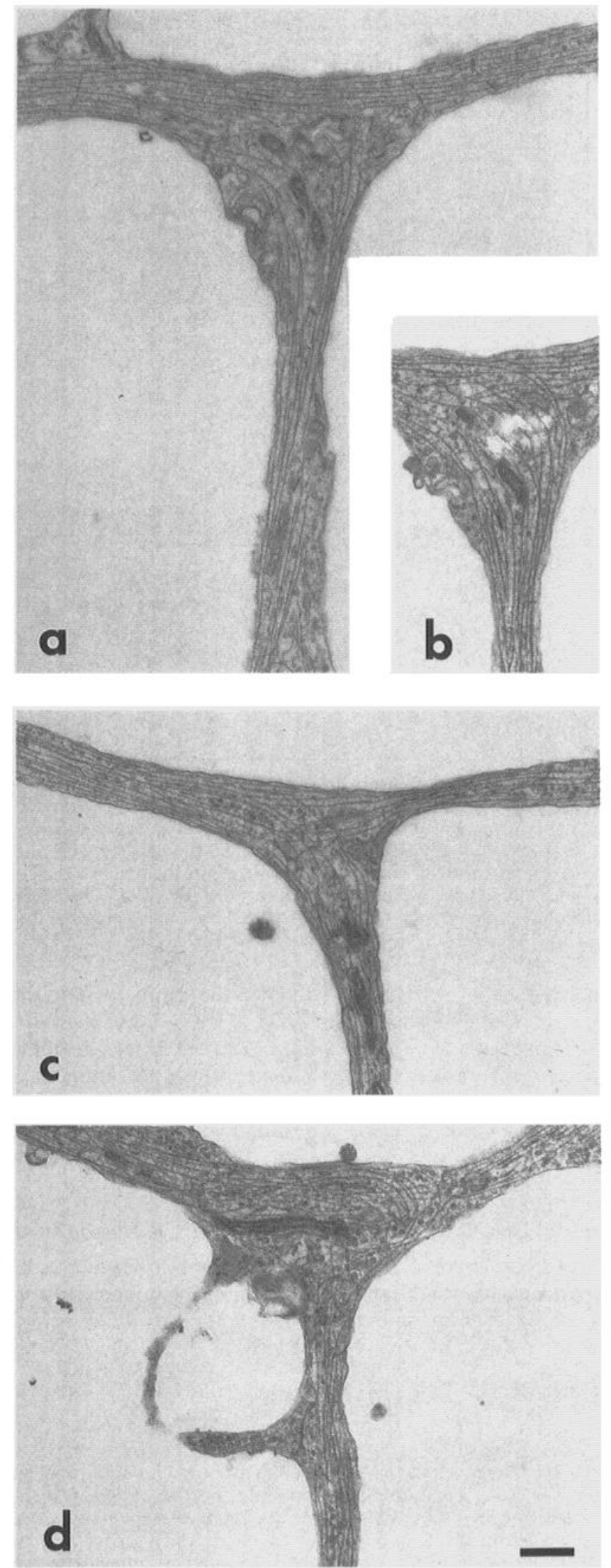

straighter MTs directed into the distal side of the parent axon. A somewhat unusual case is shown in $d$, where the curved MTs from the proximal side of the parent axon actually overshoot the branch point, extend into the distal side of the parent axon, and then curve back into the branch. Scale bar, $0.60 \mu \mathrm{m}$. 

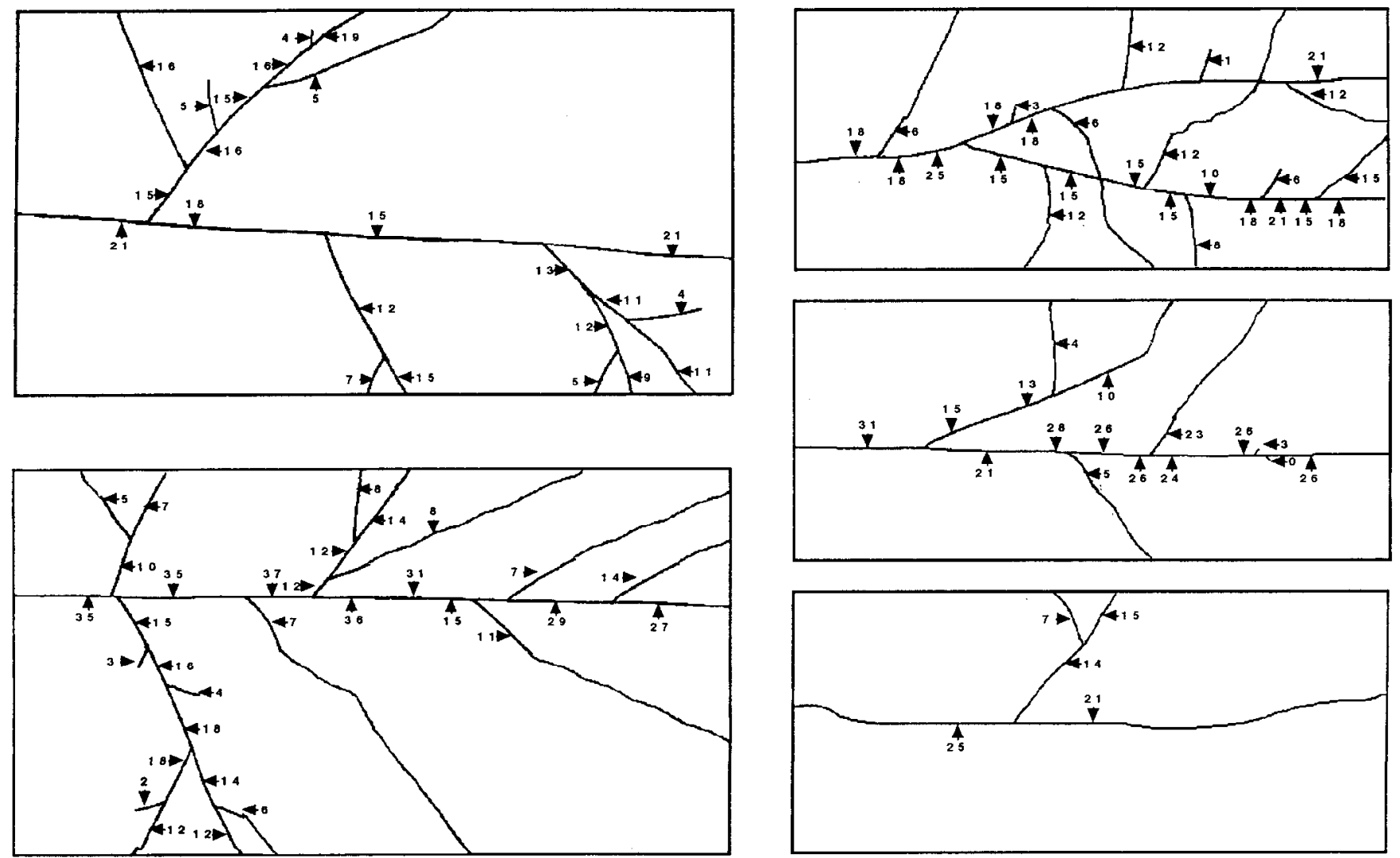

Figure 3. Schematic representations of regions of axonal arbors with estimates of the cross-sectional number of MTs at discrete sites. The procedure for obtaining these estimates is described in Materials and Methods, and data were obtained for portions of seven different axon arbors, five of which are shown here. The cross-sectional numbers of MTs within the proximal and distal sides of the parent axon were in almost all cases similar to one another, usually within a few MTs, and the number within the branch was either also similar to or somewhat lower than this value. On average, the number of MTs on the distal side of the parent axon summed with the number within the branch exceeded the number within the proximal side of the parent axon by a factor of $1.46 \pm 0.38$, indicating that the cross-sectional number of MTs increases by about $50 \%$ on average at each branch point.

another, usually within a few MTs, and the number within the branch was either also similar to or somewhat lower than this value. On average, the number of MTs on the distal side of the parent axon summed with the number within the branch exceeded the number within the proximal side of the parent axon by a factor of $1.46 \pm 0.38$, indicating that the cross-sectional number of MTs increases by about $50 \%$ on average at each branch point.

\section{Light microscopic observations of axon branch formation}

The principal goal of the present study was to elucidate the mechanisms by which the MT array of the axon increases and changes to accommodate the formation of collateral branches. For this reason, we next examined early stages of collateral branch formation. Before focusing on MTs, we explored in some detail the hehavior and morphological changes of the axon that occur as branches form. Light microscopic observations on randomly selected axon regions of varying morphology, example micrographs of which are shown in Figure 4, confirm and extend the observations of Dotti et al. (1988). As detailed in Material and Methods, regions were selected on the seventh day after plating, marked on the bottom of the dish so that they could be relocated at subsequent time points, and photographed. In most studies, the same axon regions were photographed again the following morning and thereafter at $4 \mathrm{hr}$ intervals, although in a small number of studies observations were made over time periods ranging from minutes to hours. Over $85 \%$ of all new branches that we documented formed at the sides of existing

Figure 4. Phase-contrast micrographs of regions of hippocampal neuron cultures showing the formation of branches. Separate sequences are shown in $a-c, d-g, h$ and $i$, and $j$ and $k$. The numerals on each sequence correspond to newly forming collateral branches, with the same numeral indicating the same branch within a sequence. The first micrograph in each series was taken on the seventh day after plating. The second micrograph was taken the following morning, and any subsequent micrographs were taken at $4 \mathrm{hr}$ intervals. Observation of axons at shorter time intervals indicates that multiple spurts of growth and partial or complete retraction as well as periods of inactivity all occur during these longer intervals between our micrographs (see Dotti et al., 1988). In the first sequence, $a$ shows three short processes forming along the length of an axon, $b$ shows that two of the three continued to grow into collateral branches, while the third degenerated, and $c$ shows continued growth of the branches, with the branch marked 1 extending a branch of its own. In the second sequence, $d$ shows an axon with no detectable branch formation, and $e$ shows a dramatic protrusion (marked with arrow) from the side of the axon, but carcful inspcction indicates that this is actually the terminal growth cone of a different axon growing alongside the first axon in the lower part of the panel. In addition, two newly forming collateral branches are apparent; the branch 

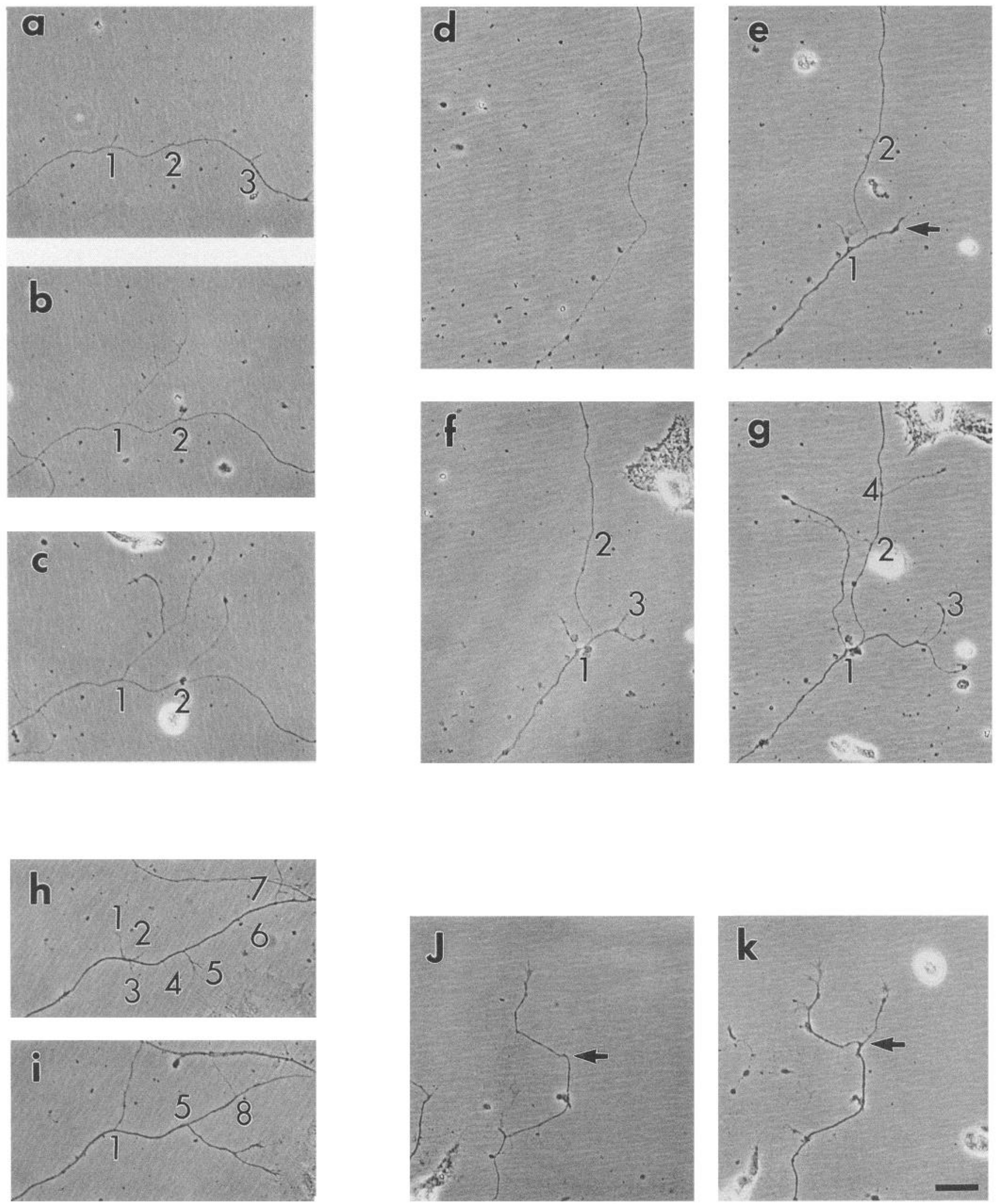

marked 2 clearly emerges from the first axon, while it is unclear whether the branch marked 1 emerges from the first or second axon. In $f$, some elongation of these branches is apparent, and a third branch has emerged near the growth cone of the second axon, and in $g$ elongation of all branches is apparent. In the third sequence, $h$ shows a region of an axon with seven different short branches starting to form, while $i$ shows that by the next morning only three of the original seven remained and grew, while the others degenerated. The fourth sequence, $j$ and $k$, illustrates that even when branch formation occurs near the growth cone, careful inspection indicates that branching usually does not occur by growth cone bifurcation, but by collateralization. The arrows indicate the site of branch formation. Scale bar, $20 \mu \mathrm{m}$. 

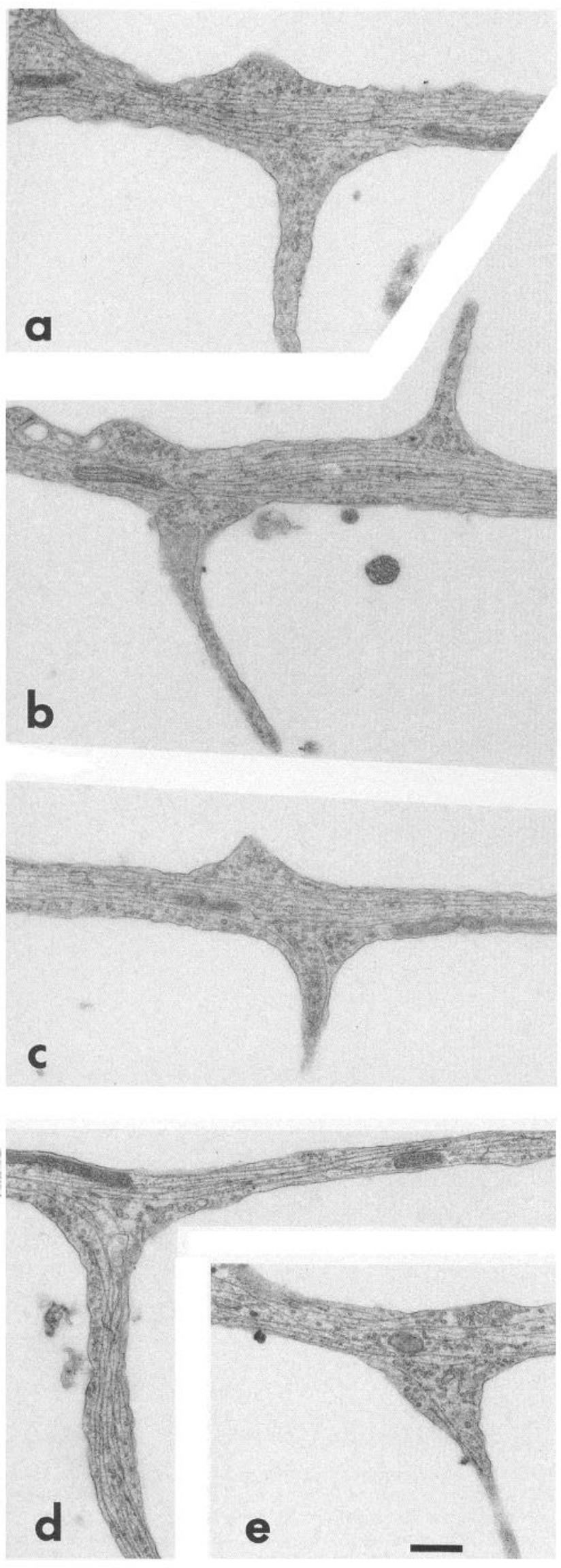

axons rather than by bifurcation of a growth cone. Although our time points were not sufficiently close to observe the most rapid dynamic events, it was our impression that collateral branches did not form first as a detectable preterminal growth cone at the side of the axon, but rather as short protrusions. In most cases, these protrusions grew at an angle of roughly $90^{\circ}$ relative to the parent axon, and this was a relatively good marker that distinguished a branch from two axons of a fascicle coming apart, the latter of which tended to occur at more acute angles (as assessed by electron microscopy; data not shown). These short branches underwent spurts of growth and retraction, and often retracted entirely. In most cases, after having grown longer than $20 \mu \mathrm{m}$, the branch no longer retracted entirely, and was committed to net growth. Rates of growth varied among branches and at different times for the same branch, with individual branches sometimes showing no growth for periods exceeding an hour. However, the rates generally fell within a range of 2$20 \mu \mathrm{m} / \mathrm{hr}$, roughly similar to that for the parent axon (see also Dotti et al., 1988).

\section{Changes in microtubule number and organization during collateral branch formation}

To assess changes in the MT array that occur during collateral branch formation, we examined the ultrastructure of axons in regions showing collateral branches of varying lengths. We first examined the short processes initially extended by the parent axon, focusing on those under $5 \mu \mathrm{m}$ in length. Most of these processes $(\approx 75 \%)$ contained no MTs, but were instead occupied by accumulations of small clear vesicles, similar to those found at synapses (Fig. $5 a-c$ ). The MT array within the parent axon in the region from which these processes grew was indistinguishable from regions from which no processes grew. That is, the MT array was continuous from the proximal into the distal side of the parent axon, with no curved MTs diverted away from the array. The remaining processes under $5 \mu \mathrm{m}$ in length $(\approx 25 \%$ ) contained small numbers of MTs (Fig. $5 b-d$ ). However, these were very sparse, with fewer than five MT profiles ap-

Figure 5. Transmission electron micrographs of newly forming collateral branches. As in Figure 2, each panel shows a branch point, with the proximal and distal sides of the parent axon directed to the left and right, respectively, and the branch directly downward. $a-c$ show branches under $5 \mu \mathrm{m}$ in length, while $d$ and $e$ show branches $10-20 \mu \mathrm{m}$ in length Most branches under $5 \mu \mathrm{m}$ in length contained no MTs, but were instead occupied by accumulations of small clear vesicles, similar to those found at synapses (see $a$ and the upper of the two processes in $b$ and $c$ ). The MT array within the parent axon in the region from which these processes grew was indistinguishable from regions from which no processes grew. That is, the MT array was continuous from the proximal into the distal side of the parent axon, with no curved MTs diverted away from the array. Other branches under $5 \mu \mathrm{m}$ in length contained small numbers of MTs (see the lower processes in $b$ and $d$ ). As in more established branches, these MTs curved in from the proximal side of the parent axon, never from the distal side, and become relatively paraxial within the process. Branches greater than $5 \mu \mathrm{m}$ in length contained greater numbers of MTs, and many of the branches 10-20 $\mu \mathrm{m}$ in length contained cross-sectional numbers of MTs similar to those observed in more established branches $(d)$. In the cases in which MTs could be found within the branch, there were also changes in the MT array of the parent axon. In addition to the curved MTs, many of the MT profiles were shorter than in other regions of the parent axon, and the integrity of the MT bundle was noticeably disturbed, with many MTs diverted away from the bundle and more variability in the spacing between MTs. These latter points are especially clear at the branch point shown in $e$. Scale bar, $0.60 \mu \mathrm{m}$. 
pearing in all sections comprising an individual process. Nevertheless, like the MTs within more established branches, these MTs curved in from the proximal side of the parent axon, never from the distal side, and become relatively paraxial within the process. Branches greater than $5 \mu \mathrm{m}$ in length contained greater numbers of MTs, and many of the branches $10-20 \mu \mathrm{m}$ in length contained cross-sectional numbers of MTs similar to those observed in more established branches (Fig. $5 d$ ). Within the parent axon, specifically at the point from which these branches emerged, there were marked alterations in the MT array. In addition to the curved MTs noted above, many of the MT profiles were shorter than in other regions of the parent axon, and the integrity of the M' bundle was disturbed, with many MTs diverted away from the bundle and more variability in the spacing between MTs (sec especially Fig. 5e).

Our premise, described in the introductory remarks, is that local increases in MT number at branch points result from the fragmentation of MTs within the parent axon. To evaluate this idea, we wished to determine during the initial stages of branch formation the numbers and lengths of MTs within the proximal and distal regions of the parent axon, and within the branch. If the MT array of the parent axon locally fragments, we would expect to find higher numbers of MTs and, at least during initial stages of branch formation, we would expect these MTs to be relatively shorter than those found elsewhere within the parent axon. We would also expect an interruption in the long lengths of MTs traversing the parent axon in the region of the branch, and a significantly higher frequency of free MT ends. To obtain the relevant data, we serially reconstructed the MT array within a $12 \mu \mathrm{m}$ collateral branch and the discrete region of the parent axon from which it grew. With regard to the latter, we reconstructed $12 \mu \mathrm{m}$ on the proximal side and $12 \mu \mathrm{m}$ on the distal side of a line bifurcating the parent axon through the branch point. These data were compared with data obtained for a region of the parent axon from which branches did not grow. The procedure for serial reconstruction is described in Materials and Methods.

Because the entire branch was encompassed in our reconstruction, we were able to determine the exact length and position of each MT contained within it (see Fig. 6). The total length of MT polymer in the branch was $128 \mu \mathrm{m}$, and this consisted of 56 MTs ranging from 0.19 to $11.4 \mu \mathrm{m}$ in length, with an average length of $2.24 \pm 2.36 \mu \mathrm{m}$. (These data included the entire lengths of MTs starting in the parent axon and curving into the branch.) Unlike the situation in the branch, many of the MTs within the parent axon extended beyond the $12 \mu \mathrm{m}$ regions of interest, precluding us from measuring their exact individual lengths. For these regions, we obtained an estimate for the average MT length by dividing the total length of polymer by the number of free MT ends (see Bray and Bunge, 1981). In the $12 \mu \mathrm{m}$ of the parent axon on the proximal side of the branch, there were 96 free MT ends in $193 \mu \mathrm{m}$ of MT polymer, indicating an average MT length of $2.0 \mu \mathrm{m}$. In the $12 \mu \mathrm{m}$ of the parent axon on the distal side of the branch, there were 70 free MT ends in $210 \mu \mathrm{m}$ of MT polymer, indicating an average MT length of $3.0 \mu \mathrm{m}$. In a typical $12 \mu \mathrm{m}$ region of the parent axon not forming branches, we scored 10 free MT ends in $271 \mu \mathrm{m}$ of total MT polymer, indicating an average MT length of $27.1 \mu \mathrm{m}$ (data not shown; see also $\mathrm{Yu}$ and Baas, 1994). Collectively, these data indicate that the discrete region of the parent axon from which the collateral branch forms contains about $20 \%$ less total polymer compared to regions of the parent axon not forming a branch, that there are 10 times as many free MT ends, and that the MTs on average are about 10 times shorter. These observations provide strong support for the view that there is a local fragmentation of MTs during collateral branch formation.

In a final serial reconstruction, we wished to determine whether notable MT length and number differences are also apparent within the parent axon at sites from which well-established branches grew. For this, we determined the lengths of MTs on the proximal and distal sides of a region of the parent axon extending a branch that was over $100 \mu \mathrm{m}$ in length. The $12 \mu \mathrm{m}$ region on the proximal side of the branch point contained 25 MT ends in $262 \mu \mathrm{m}$ of MT polymer, while the $12 \mu \mathrm{m}$ region on the distal side of the branch point contained $23 \mathrm{MT}$ ends in $228 \mu \mathrm{m}$ of polymer, for average MT lengths in these regions of 10.5 and $9.9 \mu \mathrm{m}$, respectively (data not shown). Thus, compared to the region of the parent axon not extending a branch, there were almost three times as many MT ends and the average MT length was almost three times shorter. Compared to the region of the parent axon extending the newly forming branch, however, there were almost three times fewer MT ends and the average MT length was almost three times longer. The simplest interpretation of these results is that MT fragmentation continues as the branch grows, but is less active or less focal.

\section{Microtubule age during collateral branch formation}

In a final set of studies, we wished to investigate whether a portion of the MT fragments within the parent axon is partitioned into the collateral branch as it forms. This possibility, which would require the active transport of MTs from the parent axon into the branch, contrasts with the possibility that all of the MT polymer in the newly forming branch is newly assembled. To explore this issue, we compared the age of the MT polymer within newly forming branches (ranging $\approx 5-25 \mu \mathrm{m}$ in length) with the age of the polymer within the parent axon. We reasoned that, if the MT polymer were entirely or mostly newly assembled within the branch, there would be a significantly higher proportion of newly assembled to total polymer in the branch compared to the parent axon. Alternatively, if much of the MT polymer within the branch was assembled within the parent axon and then transported into the branch, the age of the polymer in both regions should be similar. To test MT age in different regions of the axon, we extracted the cells in the presence of an MT-stabilizing buffer to remove free tubulin, and then used double-label quantitative immunofluorescence to ratio the levels of tyrosinated tubulin to $\beta$-tubulin comprising the polymer within each region. The former is an indirect indicator of newly assembled polymer and the latter is a direct indicator of total polymer (for details see Baas and Black, 1990; Baas and Ahmad, 1992; Brown et al., 1992; Ahmad et al., 1993). For each axon region of interest, the immunofluorescence images for each antibody were obtained, after which a third image was obtained, each pixel of which represents the ratio of fluorescence intensity of tyrosinated to $\beta$-tubulin within the polymer. Higher and lower ratios indicate higher and lower proportions of newly assembled polymer, respectively. The ratio image was expressed in pseudocolor, where light violet indicates the lowest ratio and deep red indicates the highest ratio (see Materials and Methods). (It should be noted that the accuracy of these determinations is best in regions where the signal is highest, but due to potential nonlinearities in the detection of fluorescence signals, may be less meaningful in regions of lower intensity, such as the rim of the cell.) 

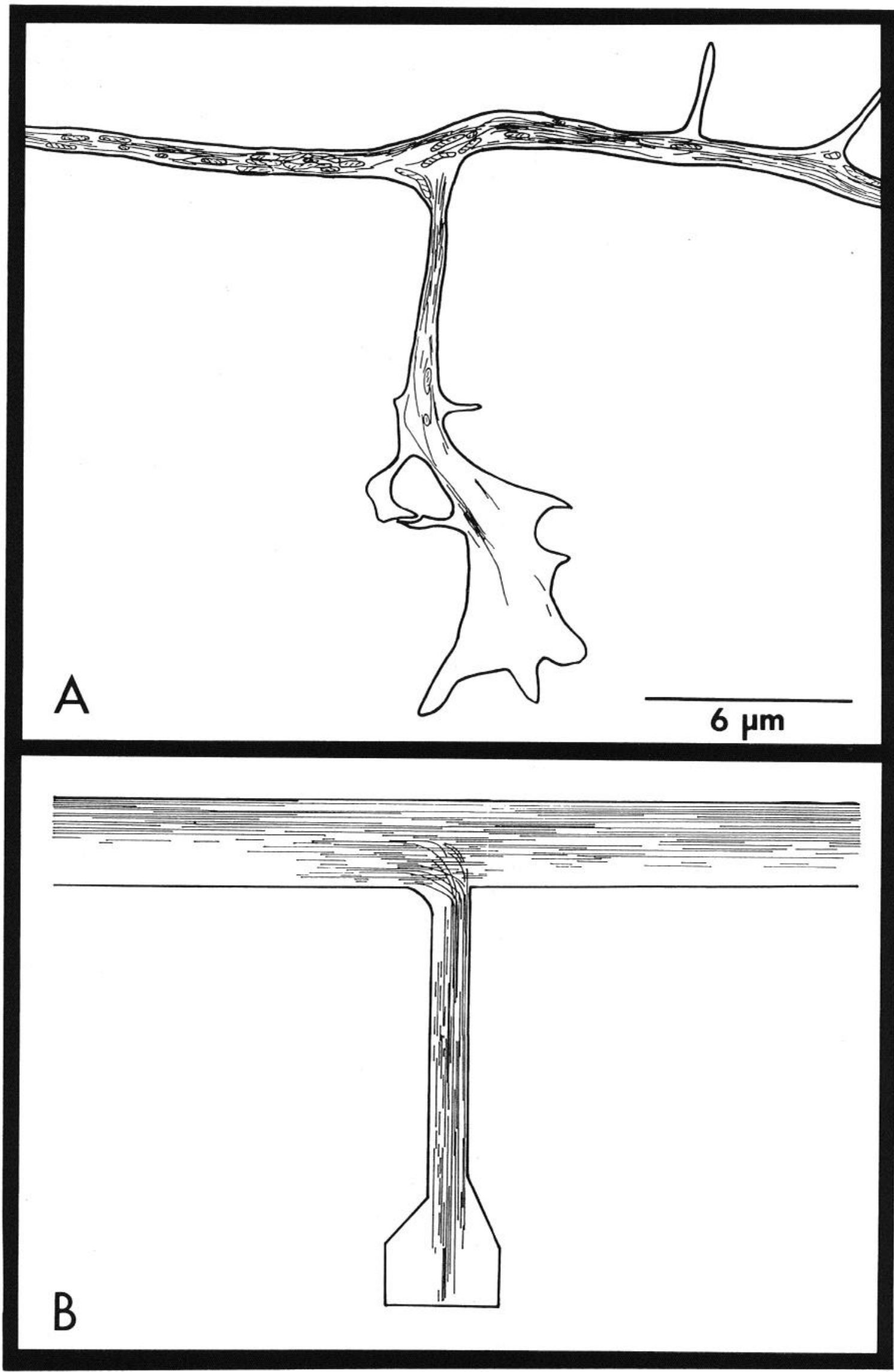

Figure 6. Serial reconstruction of the MT array within a newly formed collateral branch and the region of the parent axon from which it emerged. $A$ shows an actual tracing of one thin section. The hatched ovals are mitochondria, while the remaining linear elements shown in the tracing are MT profiles. Because individual MTs are fractionated among thin sections, observation of a single thin section does not reveal true MT lengths. 
In a previous report, Brown et al. (1992) used this approach in studies on the axons of cultured sympathetic neurons. Their work indicates that there is a particularly high ratio of newly assembled to total MT polymer in the most distal several microns of the axon contiguous with the terminal growth cone, and this conclusion has been confirmed by immunoelectron microscopy (Ahmad et al., 1993). Expressed in pseudocolor, the ratio images show red within the distal region, and violet-blue within the shaft. Our observations on cultured hippocampal neurons are essentially the same. Figure $7, a$ and $c$, shows the terminal growth cone region of a parent axon continuous with its shaft. The perimeter of the entire axon is surrounded by light violet. Within the shaft, the color is deeper purple and dark bluc with splotches of lighter blue, indicating the lower end of the scale with regard to the proportion of newly assembled polymer. Moving distally toward the growth cone, the central region of the axon takes on progressively more green and yellow color, indicating an increasingly higher proportion of newly assembled polymer. Ultimately, the central core of the axon takes on a deep red color in the most distal $\approx 60 \mu \mathrm{m}$. In direct contrast to these results, there was no green, yellow, red, or even light blue in the newly forming collateral branches, only light violet and deep purple (see Fig. $7 d-f$ ). These results, which were obtained for all of over 100 newly forming collateral branches, indicate that the MTs within the newly formed collateral branches are on average the same age as those within the parent axon, and thus support the idea that these MTs were assembled in the parent axon and then translocated into the branch.

\section{Discussion}

In the present study, we sought to understand the changes in the MT array of the axon that occur during the formation of collateral branches. In particular, we sought to understand the mechanism by which the axon accommodates the need for local increases in MT number underlying collateral branch formation, and how the pattern of MT organization within the parent axon is passed on to each collateral branch. In theory, the rapid and focal increases in MT number required for collateral branch formation could result from either de novo initiation of new MTs or by fragmentation of existing MTs. The results of our serial reconstruction analyses strongly suggest the latter. Compared to other regions of the parent axon, the discrete region surrounding a newly forming collateral branch contains a severalfold higher number of MTs, and a severalfold lower average MT length. Moreover, the number of long uninterrupted MTs traversing the parent axon decreases in the region of the branch point, with many more free MT ends present. The simplest interpretation of these findings is that prior to branch formation, MTs several times longer had traversed this region of the axon, and these long MTs were fragmented into shorter MTs as the branch formed. The other possibility, that the short MTs were assembled de novo, seems unlikely for several reasons. First, to be consistent with the present data, de novo initiation would have to have been preceded by the disassembly of the longer MTs formerly occupying the parent axon in the region of the branch. Such a shift from fewer longer MTs to higher numbers of shorter MTs is exactly opposite what would be expected based on the dynamic properties of MTs within a population (see Wordemann and Mitchison, 1994). In addition, work from our laboratory suggests that de novo initiation of MTs does not occur within the axon (Baas and Ahmad, 1992; see also Baas and Heidemann, 1986), and suppression of de novo initiation is probably important for the preservation of the uniform polarity orientation and consistent lattice structure of the MTs (see introductory remarks). Also arguing against de novo initiation are the results of our ratio-imaging studics, which show no evidence for an enrichment of newly assembled polymer at branch points or within the parent axon flanking the branch. Taking all of this into account, the most reasonable conclusion is that the local increase in MT number that occurs during the formation of a collateral branch results from the fragmentation of existing MTs within the parent axon.

The stimuli that induce MT fragmentation in the axon are unknown. One possibility is that physical factors such as lateral pulling on the MTs might induce them to break. Another possibility is that specific proteins exist in the axon that sever MTs into fragments. Centrin and katanin are two proteins that appear to have MT-severing properties in other cell types (see introductory remarks), but it is unknown at present whether these or functionally similar proteins are present in the axon. In addition, it is difficult to imagine how such proteins could be localized to branch points, in that collateral branch formation appears to be possible at virtually any time and at any site along the axon's length. An attractive possibility is that MT-severing proteins are located throughout the axon, and that cofactors regulating their activity are locally altered by physiological stimuli. For example, the activity of centrin is dependent on free calcium levels (Sanders and Salisbury, 1989), and katanin requires sufficient levels of ATP to sever MTs effectively (McNally and Vale, 1993). These same factors are among those that regulate many of the actin-associated proteins such as gelsolin (Yin and Stossel, 1979), many of which are undoubtedly involved in the reorganization of the cortical actin cytoskeleton, also required for alterations in neuronal morphology (for review, see Bamburg and Bernstein, 1991). In addition, experimental studies suggest that fragmentation and/or local reductions in MT mass are directly related to increases in the surface activity of the axon (Bray et al., 1978; Joshi et al., 1986). Thus, while the specific stimuli, proteins, and cofactors underlying MT fragmentation in the axon are unknown, it seems reasonable that there is a cascade of events in which many elements of the axoplasm undergo coordinated alterations to accommodate the formation and growth of collateral branches.

Implicit in these conclusions is that some of the short MTs resulting from fragmentation are partitioned into the branch, while the others remain to supply the distal side of the parent

$B$ is a composite schematic obtained by serially reconstructing the MT profiles appearing in all sections (see Materials and Methods and Results for more details). The total length of MT polymer in the branch, including the full length of the MTs curving in from the parent axon, was 128 $\mu \mathrm{m}$, and this consisted of $56 \mathrm{MTs}$ ranging from 0.19 to $11.4 \mu \mathrm{m}$ in length, with an average length of $2.24 \pm 2.36 \mu \mathrm{m}$. In the $12 \mu \mathrm{m}$ of the parent axon on the proximal side of the branch, there were 96 free MT ends in $193 \mu \mathrm{m}$ of MT polymer, indicating an average MT length of $2.0 \mu \mathrm{m}$. In the $12 \mu \mathrm{m}$ of the parent axon on the distal side of the branch, there were 70 free MT ends in $210 \mu \mathrm{m}$ of MT polymer, indicating an average MT length of $3.0 \mu \mathrm{m}$. These data contrast with those obtained for a typical $12 \mu \mathrm{m}$ region of the parent axon not forming branches, in which 10 free MT ends were scored in $271 \mu \mathrm{m}$ of total MT polymer, indicating an average MT length of $27.1 \mu \mathrm{m}$ (data not shown; see also Yu and Baas, 1994). Scale bar for $A, 6 \mu \mathrm{m}$; the same scale applies to the length of the schematic in $B$, but the width of the schematic is not to scale. 


\section{$\beta-T U B U L I N$}
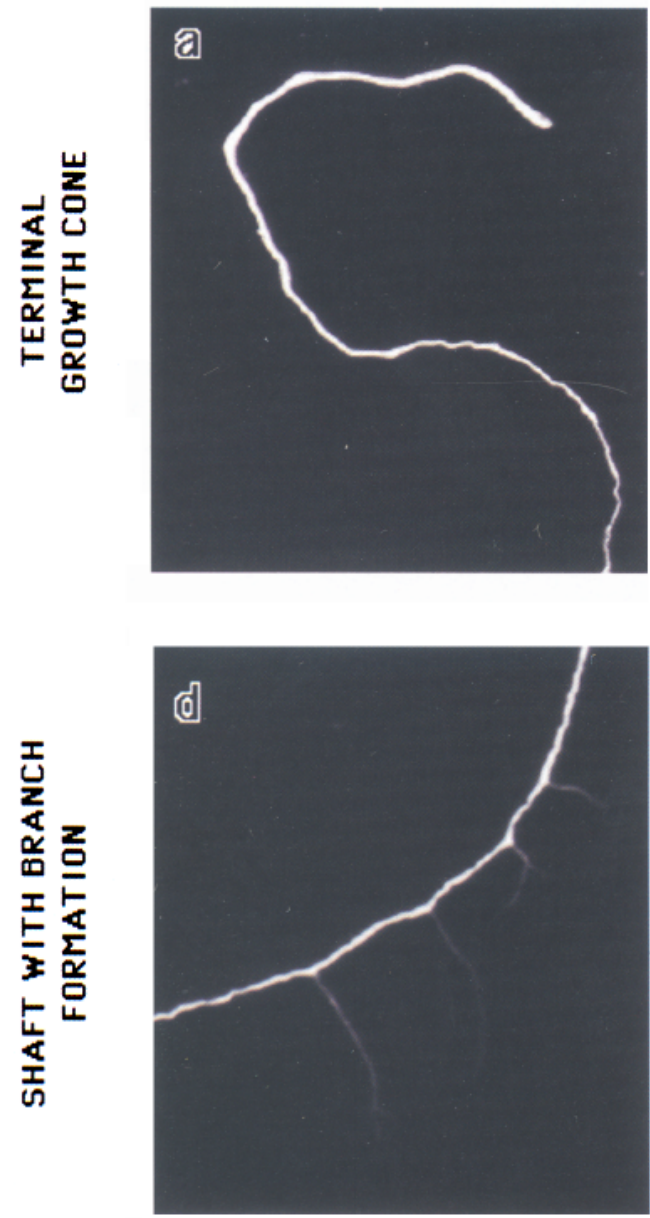

TYROSINATED

TUBULIN
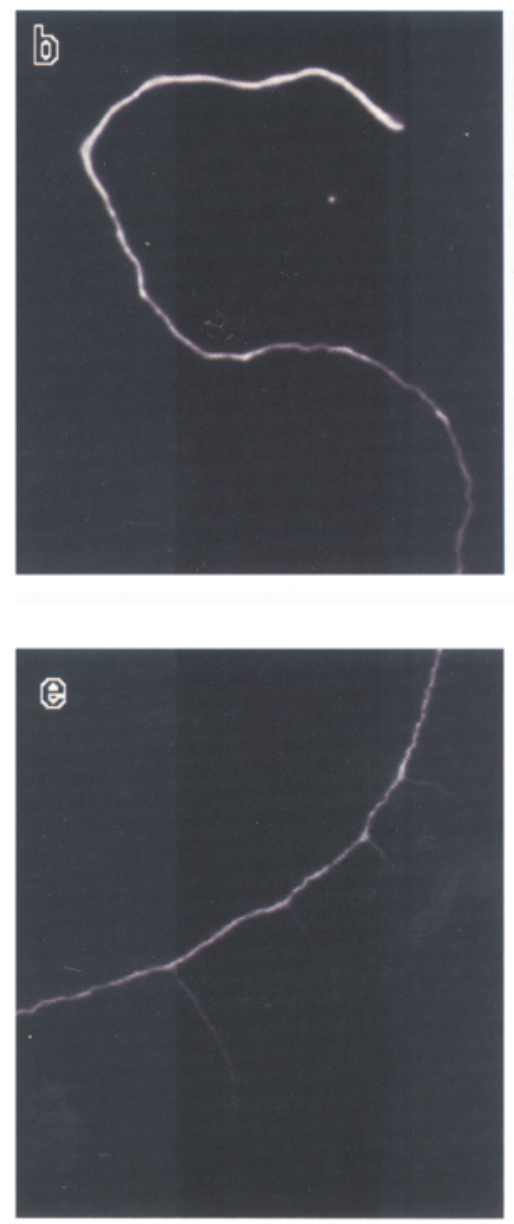

RATIO-IMAGE IN PSEUDOCOLOR
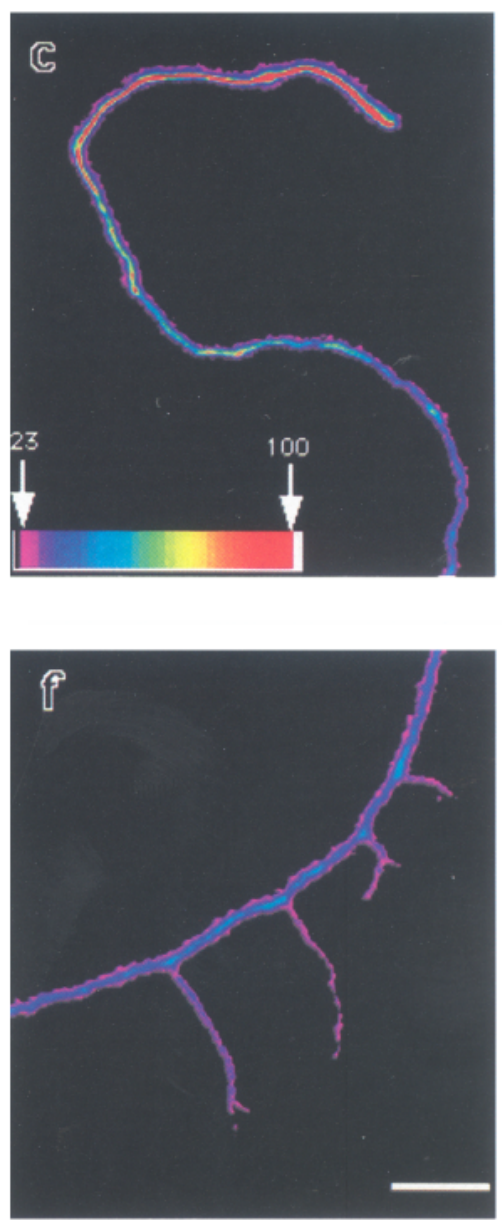

Figure 7. Quantitative immunofluorescence analyses on the relative age of the MT polymer appearing in different regions of the axon. Relative MT age was determined indirectly, by obtaining a ratio of the levels of tyrosinated to $\beta$-tubulin within MT polymer (see Materials and Methods and Results). $a$ and $d$ show immunofluorescence staining for $\beta$-tubulin in a region of the axon contiguous with the terminal growth cone and a region forming collateral branches, respectively. $b$ and $e$ show staining for tyrosinated tubulin in these same two regions, respectively. Qualitative observation indicates that relatively higher levels of tyrosinated tubulin can be found in the MTs within the distal region of the axon but not within the collateral branches, compared to the main shaft of the parent axon. However, the collateral branches also contain lower total levels of polymer, and hence it is the ratio image (tyrosinated to $\beta$ ) that provides a true indication of the proportion of newly assembled to total polymer, and hence average MT age, within each axon region. Ratio images are shown in $c$ and $f$, and are expressed in pseudocolor where light violet indicates the lowest ratio and deep red indicates the highest ratio. The perimeter of the entire axon is surrounded by light violet. Within the shaft, the color is deep purple and dark blue, with splotches of lighter blue, indicating the lower end of the scale with regard to the proportion of newly assembled polymer. Moving distally toward the growth cone $(c)$, the central region of the axon takes on progressively more green and yellow, indicating an increasingly higher proportion of newly assembled polymer. Ultimately, the central core of the axon takes on a deep red color in the most distal $\approx 60 \mu \mathrm{m}$. In direct contrast to these results, there was no green, yellow, red, or even light blue in the newly forming collateral branches, only light violet and deep purple $(f)$. Newly forming branches ranging in length from roughly 5 to $25^{\circ} \mu \mathrm{m}$ were analyzed, and the same result was obtained for all lengths. The scale indicates the range of colors, and the corresponding numerical values were obtained as indicated in Materials and Methods. Scale bar, $15 \mu \mathrm{m}$.

axon. Such a partitioning would require the active transport of MTs from one compartment to the other, and two lines of evidence suggest that such transport occurs. First, the ratioimaging analyses of MT age in different regions of the axon indicate that the MTs within the newly forming branches are assembled at essentially the same time as those within the parent axon. This result is inconsistent with the possibility that most or all of the MTs in the branch arise via new assembly. If this latter possibility were true, the proportion of newly assembled polymer in the branch would be more similar to the very high proportion of newly assembled polymer found in the MTs near the terminal growth cone. The fact that the polymer age in the newly formed branches is similar to that within the parent axon suggests that the MTs within these regions were assembled at roughly the same time, and hence that the MTs within the branches were assembled within the parent axon, and then transported into the branch. Also favoring this conclusion are observations on the MTs that curve into the branch from the parent axon. In all cases these MTs curved into the branch from the proximal side of the parent axon, never from the distal side. Given the plus-end-distal polarity orientation of the MTs, this result indicates that the MTs curving into the branch do so with their minus end in the parent axon and their plus end directed into the branch, and never with their minus end in the branch and their plus end in the parent axon. Previous data from our laboratory indicate that MT transport in the axon occurs with 
plus ends leading (Baas and Ahmad, 1993), setting up the plusend-distal polarity orientation of MTs in the parent axon, and presumably in the branches as well. The fact that MTs curve into the branch from the proximal but not the distal side of the parent axon is therefore consistent with the view that these MTs had been moving from the parent axon into the branch at the time of fixation.

Worth brief consideration is the angle at which the curved MTs bend. It seems possible that the degree to which an MT can bend may influence morphological features of branching, such as the angle at which branches grow from the parent axon. We found that early branches typically formed at about $90^{\circ}$ to the parent axon, and that with further growth the angles can become even more obtuse. Interestingly, a key morphological difference between axons and dendrites is that dendrites tend to branch at more acute angles (see Banker and Waxman, 1988; I asek, 1988). One possibility is that dendritic MTs are more rigid than axonal MTs, and this constrains the angles at which these two types of processes can branch. Compositionally, axonal and dendritic MTs differ in their complements of microtubule-associated proteins (MAPs), with the adult form of MAP-2 present only in dendrites and tau enriched in the axon (for review, see Matus, 1991). Studies in which MAP-2 is experimentally expressed in non-neuronal cells indicate that MAP-2 makes the MTs more rigid, and this rigidity assists in process formation (Edson et al., 1993). In addition, in vitro work indicates that both MAP-2 and tau reduce the flexural rigidity of MTs as assessed by their tendency to bend (Dye et al., 1993). It will be of great interest to determine whether MTs rich in tau differ from those rich in MAP-2 with regard to their flexural rigidity. Onc possibility is that tau-rich MTs are less rigid, and that this difference can explain in part the different angles at which axons and dendrites branch.

\section{Concluding remarks}

We propose the following model, based on the present results and on previous work, for the growth and perpetuation of the MT array of the axon during collateral branch formation. Initially, physiological stimuli relevant to branch formation cause local changes in regulatory factors that are as yet unidentified. These factors act on the cortical actin and membrane, causing the outgrowth of relatively short processes containing no MTs. The initial outgrowth of these branches does not require an alteration in the continuity of the underlying MT array. However, the branches are highly dynamic, often retracting entirely, and this probably relates in part to the absence of MTs within them. In the case of some of these early branches, the same or different factors cause the MTs in the parent axon to locally fragment. All of the resulting fragments inherit the 13-protofilament lattice of their predecessor MTs, and a portion of the fragments are partitioned into the newly forming collateral branch, and the others remain to supply the distal side of the parent axon. The newly formed branch is still dynamic at this point and could retract, but the branch stabilizes as it grows, and this most probably relates in part to the increase of its MT array. The partitioning of MTs requires an active transport mechanism, and this mechanism is unidirectional with regard to the polarity of the MTs, moving them exclusively with plus ends leading. In this manner, the transport properties of the MTs reestablish the uniformly plus-end-distal polarity orientation of their predecessor MTs. Once the MT array in the parent axon fragments, the cortical actin cytoskeleton and membrane become more active, facilitating the growth of the branch. During transport down the branch and the distal side of the parent axon, the MT fragments resume their dynamic exchange of tubulin subunits, resulting in a shift during transit toward smaller numbers of longer MTs. As the parent axon and the collateral branch grow, all of these events continue, including MT fragmentation events, to accommodate the steady and orderly increase of both MT arrays. In this manner, the number of MTs within the axon locally increases to accommodate collateral branch formation and growth, and the pattern of MT organization within the parent axon is passed on to each collateral branch as it forms.

\section{References}

Ahmad FJ, Pienkowski TP, Baas PW (1993) Regional differences in microtubule dynamics in the axon. J Neurosci 13:856-866.

Ahmad FJ, Joshi HC, Centonze VE, Baas PW (1994) Inhibition of microtubule nucleation at the neuronal centrosome compromises axon growth. Neuron 12:271-280.

Baas PW, Ahmad FJ (1992) The plus ends of stable microtubules are the exclusive nucleating structures for microtubules in the axon. $\mathbf{J}$ Cell Biol 116:1231-1241.

Baas PW, Ahmad FJ (1993) The transport properties of axonal microtubules establish their polarity orientation. J Cell Biol 120:14271437.

Baas PW, Black MM (1990) Individual microtubules in the axon consist of domains that differ in both composition and stability. $J$ Cell Biol 111:495-509.

Baas PW, Heidemann SR (1986) Microtubule reassembly from nucleating fragments during the regrowth of amputated neurites. J Cell Biol 103:917-927.

Baas PW, Joshi HC (1992) Gamma-tubulin distribution in the neuron: implications for the origins of neuritic microtubules. J Cell Biol 119: 171-178.

Baas PW, Deitch JS, Black MM, Banker GA (1988) Polarity orientation of microtubules in hippocampal neurons: uniformity in the axon and nonuniformity in the dendrite. Proc Natl Acad Sci USA 85:8335-8339.

Baas PW, Black MM, Banker GA (1989) Changes in microtubule polarity orientation during the development of hippocampal neurons in culture. J Cell Biol 109:3085-3094.

Bamburg JR, Bernstein BW (1991) Actin and actin-binding proteins in neurons. In: The neuronal cytoskeleton (Burgoyne RD, ed), pp $121-160$

Banker G, Goslin K (1991) Characterizing and studying neuronal cultures. In: Culturing nerve cells (Banker G, Goslin K, eds), pp 75109. Cambridge, MA: MIT Press.

Banker GA, Waxman AB (1988) Hippocampal neurons generate natural shapes in cell culture. In: Intrinsic determinants of neuronal form and function (Lasek RJ, Black MM, eds), pp 61-82. New York: Liss.

Bottenstein J (1985) Growth and differentiation of neural cells in defined media. In: Cell culture in the neurosciences (Bottenstein JE, Sato G, eds), pp 344. New York: Plenum.

Bray D, Bunge MB (1981) Serial analysis of microtubules of cultured rat sensory neurons. J Neurocytol 10:589-605.

Bray D, Thomas C, Shaw G (1978) Growth cone formation in cultures of sensory neuron. Proc Natl Acad Sci USA 75:5226-5229.

Brown A, Slaughter T, Black MM (1992) Newly assembled microtubules are concentrated in the proximal and distal regions of growing axons. J Cell Biol 119:867-882.

Burton PR, Hinkley RE, Pierson GB (1975) Tannic acid stained microtubules with 12, 13, and 15 protofilaments. J Cell Biol 65:27-233.

Dotti CG, Sullivan CA, Banker GA (1988) The establishment of polarity by hippocampal neurons in culture. J Neurosci 8:1454-1468.

Dye RB, Fink SP, Williams RC Jr (1993) Taxol-induced flexibility of microtubules and its reversal by MAP-2 and tau. J Biol Chem 268: 6847-6850.

Edson K, Weisshaar B, Matus A (1993) Actin depolymerization induces process formation in MAP2-transfected non-neuronal cells. Development 117:689-700.

Febvre-Chavalier C, Fabvre J (1992) Microtubule disassembly in vivo: intercalary destabilization and breakdown of microtubules in the heliozoan Actinocoryne contractilis. J Cell Biol 118:585-594. 
Goslin K, Banker G (1991) Rat hippocampal neurons in low-density culture. In: Culturing nerve cells (Banker G, Goslin K, eds), pp 251281. Cambridge, MA: MIT Press.

Heidemann SR, Landers JM, Hamborg MA (1981) Polarity orientation of axonal microtubules. J Cell Biol 91:661-665.

Joshi HC, Baas PW (1993) A new perspective on microtubules and axon growth. J Cell Biol 121:1191-1196.

Joshi HC, Baas P, Chu DT, Heidemann SR (1986) The cytoskeleton of neurites after microtubule depolymerization. Exp Cell Res 163: 233-245.

Kilmartin JV, Wright B, Milstein C (1982) Rat monoclonal antitubulin antibodies derived by using a new nonsecreting rat cell line. $J$ Cell Biol 93:576-582

Lasek RJ (1988) Studying the intrinsic determinants of neuronal form and function. In: Intrinsic determinants of neuronal form and function (Lasck RJ, Black MM, eds), pp 1-58. New York: Liss.

Lyser KM (1968) An electron microscopic study of centrioles of differentiating neuroblasts. J Embryol Exp Morphol 20:343-354.

Matus A (1991) Microtubule-associated proteins and neuronal morphogenesis. J Cell Sci [Suppl] 15:61-67.

McNally FJ, Vale RD (1993) Identification of katanin, an ATPase that severs and disassembles stable microtubules. Cell 75:419-429.

O'Leary DDM, Terashima T (1988) Cortical axons branch to multiple subcortical targets by interstitial axon budding: implications for target recognition and "waiting periods." Neuron 1:901-910.

Sanders MA, Salisbury JL (1989) Centrin-mediated microtubule sev- ering during flagellar excision in Chlamydomonas reinhardii. J Cell Biol 108:1751-1760.

Shiina N, Gotoh Y, Nishida E (1992) A novel homo-oligomeric protein responsible for an MPF-dependent microtubule-severing activity. EMBO J 11:4723-4731.

Tilney LG, Bryan J, Bush DJ, Fujiwara K, Mooseker MS, Murphy DB (1973) Microtubules: evidence for 13 protofilaments. J Cell Biol 59: 267-275.

Vale RD (1991) Severing of stable microtubules by mitotically activated protein in Xenopus egg extracts. Cell 64:827-839.

Wheland J, Willingham MC, Sandoval IV (1983) A rat monoclonal antibody reacting specifically with the tyrosinated form of $\alpha$-tubulin. I. Biochemical characterization, effects on microtubule polymerization in vitro, and microtubule polymerization and organization in vivo. J Cell Biol 97:1476-1490.

Wordcman L, Mitchison TJ (1994) Dynamics of microtubulc asscmbly in vivo. In: Microtubules (Hyams JS, Lloyd CW, eds), pp $287-$ 301.

Yin HL, Stossel TP (1979) Control of cytoplasmic actin gel-sol transformation by gelsolin, a calcium-dependent regulatory protein. Nature 281:583-586.

Yu W, Baas PW (1994) Changes in microtubule number and length during axon differentiation. J Neurosci 14:2818-2829.

Yu W, Centonze VE, Ahmad FJ, Baas PW (1993) Microtubule nucleation and release from the neuronal centrosome. J Cell Biol 122: 349-359. 